\title{
Ring-Type Erbium-Doped Antiresonant Reflecting Optical Waveguide Amplifier Analysis and Design
}

\author{
David Benedicto and Juan A. Vallés
}

\begin{abstract}
Erbium-doped antiresonant reflecting optical waveguides (ARROWs) allow combining wavelength selective guiding due to their attractive spectral versatility with an active operation. In this letter, the analysis and design of a ring-type erbiumdoped ARROW amplifier is presented. The influence of the involved passive and active parameters (ring thickness and diameter, refractive index variation, pump and signal wavelengths, $\mathrm{Er}^{3+}$-ion concentration, and input pump power) on the spectral response of the structure and the optical power propagation losses and on the amplifier performance is numerically analysed. The opposite influence of the ring diameter on the optical power confinement and on the pump power density causes the existence of a diameter value that maximizes the amplifier net gain. For a cladding refractive index of 1.4 and moderate index variations, $\Delta n=0.2-0.4$, the optimum ring diameter is in the range of $20 \mu \mathrm{m}$. To compensate signal confinement losses (a few $\mathrm{dB} / \mathrm{cm}$ ), high erbium concentrations $\left(\sim 1 \times 10^{26} \mathrm{ion} / \mathrm{m}^{3}\right)$ are required.
\end{abstract}

Index Terms-ARROW, $\mathbf{E r}^{3+}$-doping, optical amplifier, optimized design.

\section{INTRODUCTION}

$\mathbf{M}$ ICROSTRUCTURED optical fibres (MOFs) are widely used nowadays in many areas of science and technology [1], [2]. Their cross section consists of a central core surrounded by an area with multiple periodic inclusions. Basically, MOFs can be divided into two groups depending on their guiding mechanism. On one hand, index-guiding microstructured optical fibres (IG-MOFs) have a core with a higher refractive index than the cladding, so light is guided in the core due to a particular version of total internal reflection. On the other hand, photonic bandgap microstructured optical fibres (PBG-MOFs) have a core area with a lower refractive index than the cladding, so there cannot be total reflection, and light is confined due to the creation of a photonic bandgap that prevents light from escaping to the periodic cladding within certain wavelength ranges.

It has been shown that in the case of PBG-MOFs, there is a regime in which the positions of the spectral minima are mainly determined by the individual properties of the higher index inclusions, rather than by their number or position [3].

Manuscript received June 12, 2018; revised August 24, 2018; accepted October 2, 2018. This work was supported in part by the Spanish Ministry of Economy and Competitiveness under Project TEC2014-52642-C2, in part by the Diputación General de Aragón, and in part by the European Social Fund. (Corresponding author: Juan A. Vallés.)

D. Benedicto is with the Applied Physics Department, University of Zaragoza, 50009 Zaragoza, Spain (e-mail: davidbene_92@hotmail.com).

J. A. Vallés is with the Applied Physics Department, Aragón Institute of Engineering Research, University of Zaragoza, 50009 Zaragoza, Spain (e-mail: juanval@unizar.es).

Digital Object Identifier 10.1109/LPT.2018.2876571

This guiding mechanism can be described by an antiresonant reflecting optical waveguide (ARROW) model [4]. In this model if the light reaching the inclusion is on transverse resonance, it escapes from the core. On the contrary, when in an antiresonant condition, the light is reflected back into the core and it results guided. ARROW-type waveguides offer some peculiarities compared to standard PBG-MOF structures [5]. ARROWs give the possibility of designing PBG-MOFs exhibiting attractive spectral properties without the necessity of a strict periodicity in the structure, being often used for guiding light in liquids or gases [6]. Moreover, ARROW-type MOFs enable a more flexible tuning range compared with grating-based devices, which is one of the several advantages of using it to design tunable devices [7] or for fibre sensing applications [8].

In the literature examples of PCFs doped with erbium and/or ytterbium and with interesting properties for active fibre and all-fibre amplifier devices can be found [9], and different studies have been carried out on this technology in order to improve the performance of rare earth doped fibre amplifiers and lasers [10]. Following this idea we propose a model for an erbium doped ARROW capable of guiding both the wavelength of the peak of emission of the erbium ion $\mathrm{Er}^{3+}$ and the pump wavelength. The optimization of this device will allow us to open the ARROW mechanism into the huge range of possibilities related with erbium-doped fibre amplifiers (EDFAs).

In this letter we present a procedure for the design of a ring-type erbium-doped ARROW amplifier. First, in section 2, the passive response of the structure is analyzed and the parameters for a low-loss confinement of both pump and signal wavelengths of the ARROW amplifier are determined. Then, in section 3 by calculating the optical powers propagation along the erbium-doped structure the design parameters for an optimum ring-type ARROW amplifying performance are obtained.

\section{PAssive BehaVior of A Ring-Type ARrow}

Antiresonant reflecting optical waveguides can guide light with low loss due to the thin-layer interference principle. In this phenomenon light waves reflected from a thin layer interfere with one another and depending on the relative phase difference can produce constructive or destructive interference.

In order to illustrate the guiding mechanism consider light waves propagating in the planar structure shown in figure 1(a). For a given wavelength when the thickness of the darker higher 


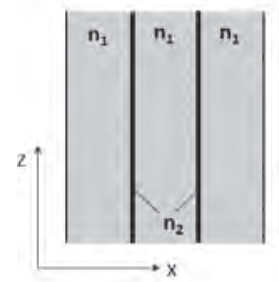

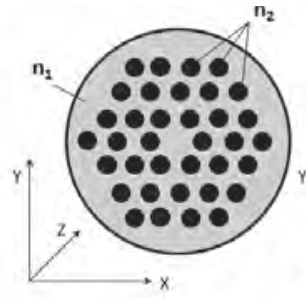

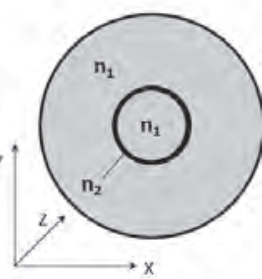

c)
Fig. 1. Scheme of (a) a planar ARROW structure, (b) a PBG-MOF and (c) a ring-type ARROW MOF.

index $\left(\mathrm{n}_{2}\right)$ strips is such that the reflected waves are in phase, light can be confined in the central low-index $\left(\mathrm{n}_{1}\right)$ region and propagated along the $z$ direction, resulting in a guided spectrum maximum. On the contrary, reflection minima will occur when the light waves that interfere are transmitted across the $\mathrm{n}_{2}$ strips and then a guided spectrum minimum occurs. The optical modes of the structure will be leaky, but it is possible to achieve low propagation losses as in a high quality factor Fabry-Perot reflector.

Among the different possible 3D ARROW structures a lower index core surrounded by higher index inclusions is the one mostly found in the literature [3], [7], [8] (see Fig.1(b)). Another interesting option is the ring-type ARROW which is represented in Fig.1(c). The mechanism governing the spectral behavior of a ring-type ARROW structure is analogous to that of the planar geometry presented in figure 1(a). This similarity has already been reported for the case of the high index inclusions forming a typical MOF transverse structure [3], [8]. To calculate the structure spectral response we have used a commercial software that allows us to compute the field profile and complex effective refractive index of the ARROW structure leaky modes (BeamPROP - RSoft CAD). The correlation method is used in our simulation together with the Perfectly Matched layer (PML) for the boundary conditions. The size of the computational window is $29 \mu \mathrm{m} \times 29 \mu \mathrm{m}$, the transversal grid size was $0.02 \mu \mathrm{m} \times 0.02 \mu \mathrm{m}$ whereas for parameter sweeps the transversal grid size is $0.1 \mu \mathrm{m} \times 0.1 \mu \mathrm{m}$ so that error was sufficiently low and the computation time was affordable. The longitudinal grid size has been varied in the range 0.1-0.4 $\mu \mathrm{m}$ and the excitation source is a Gaussian. Passive losses can be obtained from the imaginary part of the effective index of the leaky propagation mode.

In order to illustrate the spectral behavior of this kind of structures we calculate the guided spectrum after a $1 \mathrm{~cm}$ propagation of a ring-type ARROW with a cladding refractive index $\mathrm{n}_{1}=1.4$, a refractive index variation $\Delta \mathrm{n}=0.4$, a ring inner diameter $\mathrm{D}=18 \mu \mathrm{m}$ and a ring thickness $\mathrm{d}=1.07 \mu \mathrm{m}$. For this range of values the influence of the ring diameter on the spectral response is relatively small. In figure 2 the computed guided spectrum is shown for the wavelength region of interest for the erbium-doped amplifier. In the near infrared range near zero minima are obtained for $0,807 \mu \mathrm{m}$ and $1.21 \mu \mathrm{m}$ whereas the relative maxima are found for $722 \mu \mathrm{m}$, $957 \mu \mathrm{m}$ and $1543 \mu \mathrm{m}$. For these maxima the normalized propagated power after $1 \mathrm{~cm}$ decreases with the wavelength and is $0.989,0.971$ and 0.864 , respectively. Wavelengths for

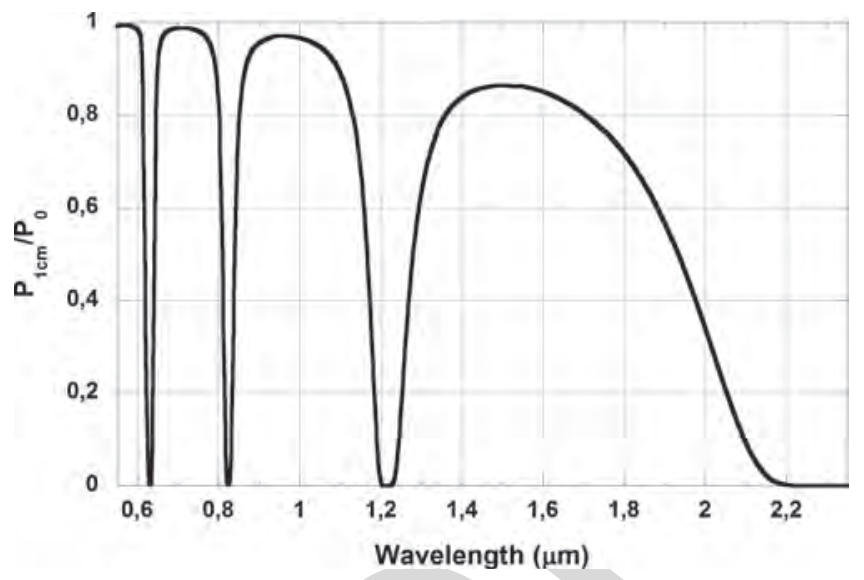

Fig. 2. Guided spectrum after a $1 \mathrm{~cm}$ propagation for a ring-type ARROW with $\mathrm{n}_{1}=1.4, \mathrm{n}_{2}=1.8, \mathrm{~d}=1.07 \mu \mathrm{m}$ and $\mathrm{D}=18 \mu \mathrm{m}$.

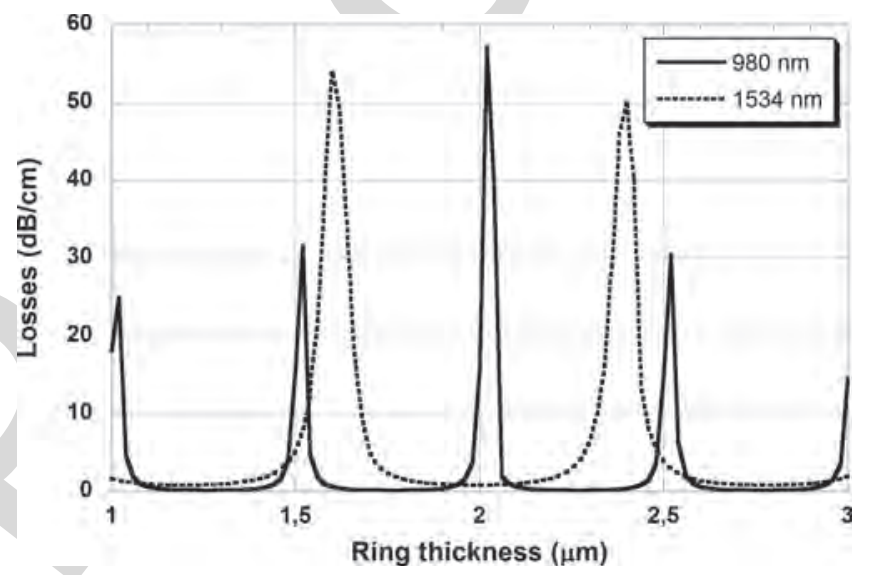

Fig. 3. Propagation losses for the pump $(980 \mathrm{~nm})$ and the signal $(1534 \mathrm{~nm})$ wavelengths as a function of the ring thickness, in a ring-type ARROW with $\mathrm{n}_{1}=1.4, \mathrm{n}_{2}=1.7$ and $\mathrm{D}=20 \mu \mathrm{m}$ parameters.

the guided spectrum minima in figure 2 can be estimated using the resonant condition equation $\lambda=(2 d / m) \sqrt{n_{2}^{2}-n_{1}^{2}}$ (where $m$ is an integer) with a less than $5 \%$ error.

Since our aim is to design an erbium doped amplifier we select the geometrical parameters of the structure in such a way that both pump and signal wavelengths $(980 \mathrm{~nm}$ and $1534 \mathrm{~nm}$ respectively) approximately correspond to propagation maxima. In order to do this, we need to find a ring thickness that fulfils both conditions for a given index variation.

In figure 3 losses at the two wavelengths (980 and $1534 \mathrm{~nm}$ ) are plotted as a function of the ring thickness for an index variation of $\Delta \mathrm{n}=0.3$. We choose the region for the smallest thickness (around $1.2 \mu \mathrm{m}$ ), where signal and pump losses are both near a minimum, $0.17 \mathrm{~dB} / \mathrm{cm}$ and $0.81 \mathrm{~dB} / \mathrm{cm}$, respectively. By changing the refractive index variation, it is found that the ring thickness that presents low loss propagation for both wavelengths depends inversely on $\Delta \mathrm{n}$. This dependence is shown in figure 4. From now on and when designing the ARROW amplifier we consider the ring thickness obtained following this procedure for each $\Delta \mathrm{n}$ as the optimum ring thickness. 


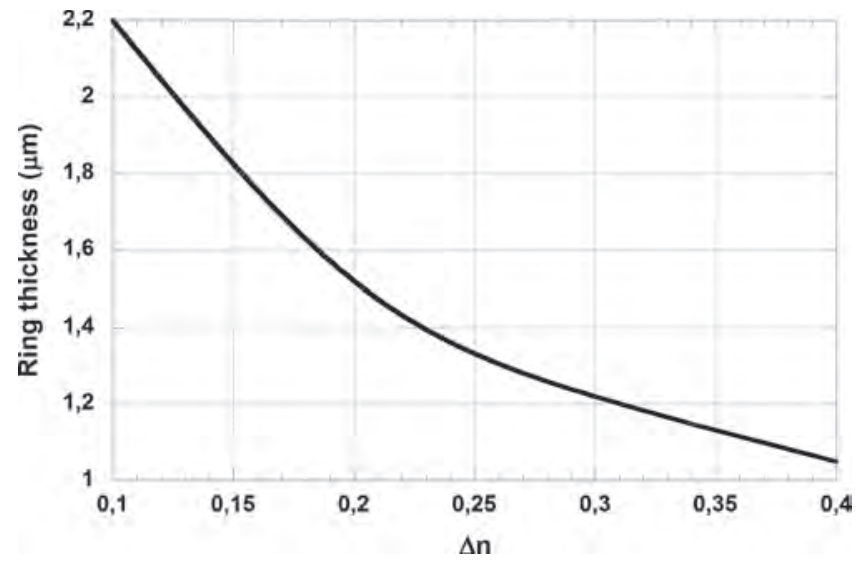

Fig. 4. Smallest ring thickness that presents low loss propagation for both pump $(980 \mathrm{~nm})$ and signal $(1534 \mathrm{~nm})$ wavelengths as a function of the refractive index variation, $\Delta \mathrm{n}$.

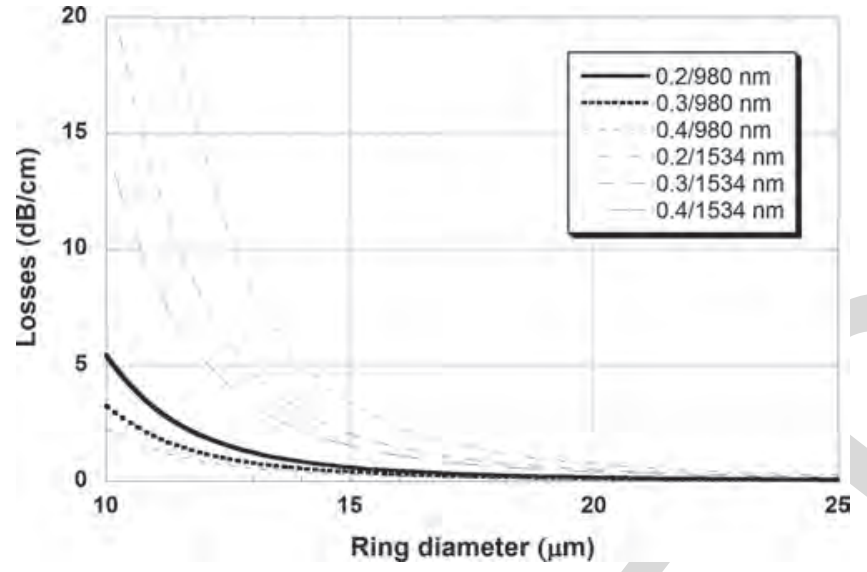

Fig. 5. Passive losses for the pump $(980 \mathrm{~nm})$ and the signal $(1534 \mathrm{~nm})$ wavelengths as a function of the ring diameter for three different values of refractive index variation $\Delta \mathrm{n}$ and optimum ring thickness.

Although we have asserted that the ring diameter for $\mathrm{D} \sim 20 \mu \mathrm{m}$ has no significant impact on the spectral response, it strongly affects the passive losses of the structure. In figure 5 losses are plotted both for the pump $(980 \mathrm{~nm})$ and the signal $(1534 \mathrm{~nm})$ wavelengths as a function of the ring diameter and for three different values of the refractive index variation. For each value of $\Delta \mathrm{n}$, the ring thickness is that in figure 4, the previously defined as optimum ring thickness. For shorter wavelengths (pump) and higher refractive index variations the power confinement is higher and subsequently the losses are smaller.

As the final goal of this study is to design an erbium doped ARROW amplifier, we need our signal losses to be at least lower than the achievable gain, which depends mostly on the $\mathrm{Er}^{3+}$ ion concentration and the available input pump power. A small ring diameter may entail too high losses to contemplate any practical active device.

\section{ERbium-Doped Ring-Type Arrow Amplifier}

In order to calculate the gain of the erbium doped ARROW amplifier and optimize its design, we have used a homemade computer code which evaluates the power propagation

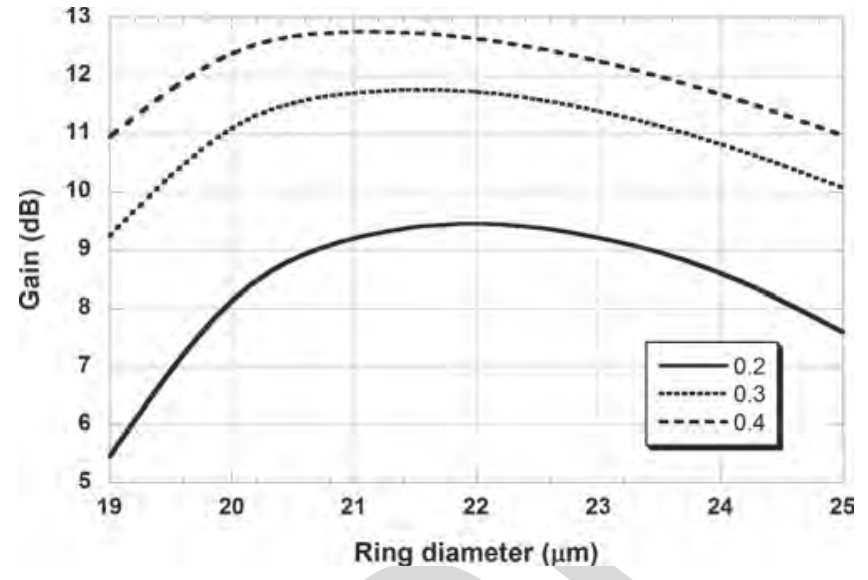

Fig. 6. Net gain of the erbium-doped ring-type ARROW amplifier as a function of the ring diameter for three different index variations with its respective optimized ring thickness. The amplifier length is $10 \mathrm{~cm}$, the input pump power is $\mathrm{P}_{\mathrm{p}}=200 \mathrm{~mW}$, the $\mathrm{Er}^{3+}$-ion concentration is $1 \times 10^{26}$ ions $/ \mathrm{m}^{3}$ and signal wavelength is $1534 \mathrm{~nm}$.

equations of the structure modes coupled to the rate equations of the involved active ions. The well-known models commonly used for step index fibers can also be used to describe the signal and pump evolution, together with the amplified spontaneous emission (ASE), along the erbium-doped ARROW structure [11]. Since to compensate losses and achieve positive net gain we will be dealing with a highly doped waveguide it is mandatory to take into account the nonradiative concentrationdependent upconversion processes with a negative impact on the pump efficiency [12]. This model can be used to design optical amplifiers, in particular erbium doped silica MOF once the necessary input spectroscopic parameters are known. These parameters, if possible, must preferably be obtained experimentally [13].

The geometrical parameter that has a greater influence on the ARROW amplifier gain is the ring diameter. As we have shown in section 2, passive losses strongly decrease as a function of the ring diameter. However, on the opposite the larger the ring diameter, the larger the mode area and, subsequently, the lower the pump power density.

In Fig. 6 the net gain of a ring-type erbium-doped ARROW amplifier is plotted as a function of the ring diameter for three values of the index variation. For each $\Delta \mathrm{n}$ the corresponding optimum ring thickness is used (see section 2). In figure 6 the amplifier length is $10 \mathrm{~cm}$, the input pump power is $P_{p}=$ $200 \mathrm{~mW}$, the $\mathrm{Er}^{3+}$-ion concentration is $1 \times 10^{26}$ ions $/ \mathrm{m}^{3}$ and signal wavelength is $1534 \mathrm{~nm}$. From figure 6 it is clear that for each $\Delta \mathrm{n}$ there is a ring diameter (in the $20 \mu \mathrm{m}$ range) that maximizes the amplifier net gain and the optimum diameter slightly increases as the refractive index variation decreases. The achievable ARROW amplifier net gain also decreases with $\Delta \mathrm{n}$ but, as we have mentioned when analyzing the impact of the size of the ring on the passive losses, this can be attributed to the influence of the signal confinement, which is lower for smaller refractive index differences. Achievable gain values for larger $\Delta \mathrm{n}$ are comparable to that of a standard single-mode EDFA with the same length, dopant concentration and pump power $(15.9 \mathrm{~dB})$. 


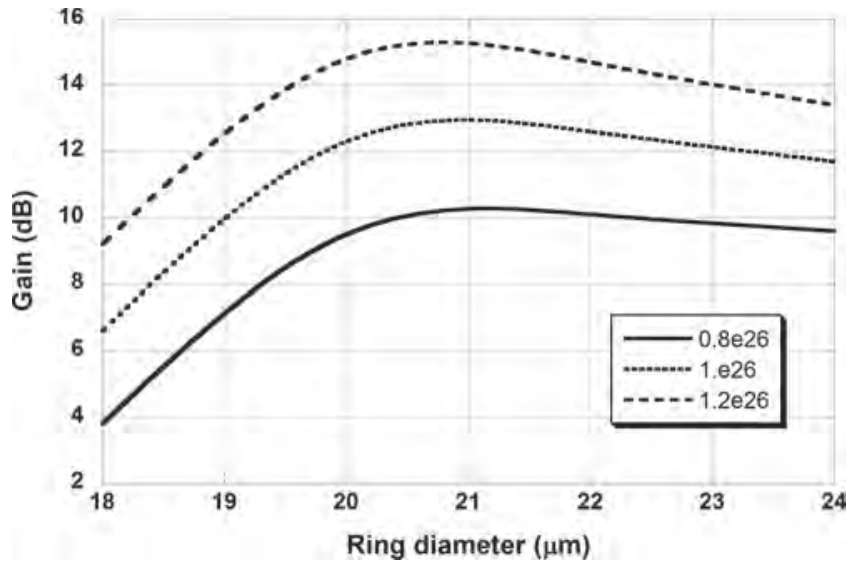

Fig. 7. Net gain of the erbium-doped ring-type ARROW amplifier as a function of the ring diameter for three different dopant concentrations with $\Delta \mathrm{n}=0.4$. The ring thickness is $1.07 \mu \mathrm{m}$, the amplifier length is $10 \mathrm{~cm}$, the input pump power is $P_{p}=200 \mathrm{~mW}$ and signal wavelength is $1534 \mathrm{~nm}$.

As shown in figure 2 the guided spectrum does not change importantly in the $1.5 \mu \mathrm{m}$ region, therefore the spectral gain response will be basically determined by erbium emission/absorption cross section distributions in the host material as in other erbium-doped amplifying structures.

Gain dependence on dopant concentration is plotted in figure 7 for $\Delta \mathrm{n}=0.4$ and same amplifier length, pump power and signal wavelength than in figure 6 . For higher concentrations the optimum ring diameter slightly decreases.

The definite erbium doping level for optimum performance will depend on the ring diameter, refractive index variation and the available pump power. Nevertheless, in practice, in order to compensate losses a high-doping level becomes indispensable.

As can be concluded from figures 3, 6 and 7, compared to the effects of ring diameter or dopant concentration variations ring thickness is the most critical parameter in what regards fabrication tolerances. Manufacturing inaccuracies of only $5 \%$ would drastically increase either pump or signal losses preventing practical amplification.

\section{CONCLUSIONS}

In this study we have demonstrated that it is possible to design a 3D ring-type ARROW waveguide structure in which both the amplified signal and pump power are low-loss guided, thus allowing the design of an erbium-doped ringtype ARROW amplifier. Using the antiresonance requisite a ring thickness that allows a low loss propagation of both pump and signal wavelengths can be determined. Then, the optimum ring diameter (that basically determines confinement losses and the pump power density) has to be obtained to maximize the ARROW amplifier net gain.
Although we have focused on a ring-type ARROW there are other transversal refractive index distributions that could offer adequate low-loss propagation. By adding new concentric rings to the structure passive losses could be further reduced, but the fabrication difficulty increases. Instead of higherindex rings individual inclusions forming different geometries could be added. An optimization procedure similar to the one followed in this letter would provide a good understanding of the influence of each parameter on the passive and active performance of the structure.

Moreover, a deeper study on its modal behavior could enlighten its use as a Large Mode Area (LMA) structure for high power waveguide lasers. Finally, if the refractive index of the high-index ring is somehow altered and the resonance condition is modified the amplifier output would accordingly change and the structure could be used as a tunable amplifier or as a waveguide sensor.

\section{REFERENCES}

[1] Z. Liu, H.-Y. Tam, L. Htein, M.-L. V. Tse, and C. Lu, "Microstructured optical fiber sensors," J. Lightw. Technol., vol. 35, no. 16, pp. 3425-3439, Aug. 15, 2017.

[2] S. M. G. Rodrigues, M. Facão, and M. F. S. Ferreira, "Supercontinuum generation in chalcogenide layered spiral microstructured optical fiber," J. Nonlinear Opt. Phys. Mater, vol. 26, no. 4, p. 1750049, 2017.

[3] T. Lewi, J. Ofek, and A. Katzir, "Antiresonant reflecting microstructured optical fibers for the mid-infrared," Appl. Phys. Lett., vol. 102, no. 10 p. 101104, 2013.

[4] M. A. Duguay, Y. Kokubun, and T. L. Koch, "Antiresonant reflecting optical waveguides in $\mathrm{SiO}_{2}$-Si multilayer structures," App. Phys. Lett., vol. 49, no. 1, pp. 13-15, May 1986.

[5] N. M. Litchinitser, A. K. Abeeluck, C. Headley, and B. J. Eggleton, "Antiresonant reflecting photonic crystal optical waveguides," Opt. Lett., vol. 27 , no. 18 , pp. 1592-1594, Sep. 2002

[6] H. Schmidt, D. Yin, D. W. Deamer, J. P. Barber, and A. R. Hawkins, "Integrated ARROW waveguides for gas/liquid sensing," Proc. SPIE, vol. 5515, pp. 67-81, Oct. 2004.

[7] N. M. Litchinitser et al., "Application of an ARROW model for designing tunable photonic devices," Opt. Exp., vol. 12, no. 8, pp. $1540-1550,2004$.

[8] N. M. Litchinitser and E. Poliakov, "Antiresonant guiding microstructured optical fibers for sensing applications," Appl. Phys. B, Lasers Opt., vol. 81, nos. 2-3, pp. 347-351, Jul. 2005.

[9] K. Mondal and P. R. Chaudhuri, "Designing high performance $\mathrm{Er}^{+3}$ doped fiber amplifier in triangular-lattice photonic crystal fiber host towards higher gain, low splice loss," Opt. Laser Technol., vol. 43, no. 8 , pp. 1436-1441, Nov. 2011.

[10] J. A. Sanchez-Marti, J. M. A. Abenia, M. A. Rebolledo, an M. V. Andres, "Amplifiers and lasers based on erbium-doped photonic crystal fiber: Simulation and experiments," IEEE J. Quantum Electron., vol. 48, no. 3, pp. 338-344, Mar. 2012.

[11] E. Desurvire, Erbium-Doped Fiber Amplifiers: Principles And Applications. Hoboken, NJ, USA. Wiley. 2002.

[12] J. A. Valles, V. Berdejo, M. Á Rebolledo, A. Diez, J. A. Sanchez-Martin, and M. V. Andres, "Dynamic characterization of upconversion in highly Er-doped silica photonic crystal fibers," IEEE J. Quantum Electron., vol. 46, no. 8, pp. 1015-1022, Aug. 2012.

[13] J. A. Sanchez-Martin, M. Á. Rebolledo, J. M. Alvarez, J. A. Valles, A. Diez, and M. V. Andres, "Erbium-doped-silica photonic crystal fiber characterization method: Description and experimental check," IEEE J. Quantum Electron., vol. 46, no. 8, pp. 1145-1152, Aug. 2010. 


\section{AUTHOR QUERIES}

\section{AUTHOR PLEASE ANSWER ALL QUERIES}

PLEASE NOTE: We cannot accept new source files as corrections for your paper. If possible, please annotate the PDF proof we have sent you with your corrections and upload it via the Author Gateway. Alternatively, you may send us your corrections in list format. You may also upload revised graphics via the Author Gateway.

AQ:1 = The ORCID provided for Juan A. Vallés was not correct and has been removed. There is no name on the ORCID webpage. Please provide a correct one.

AQ:2 = Author: Please confirm or add details for any funding or financial support for the research of this article.

AQ:3 = Please confirm the volume no. for ref. [6]. 